\title{
First HIBP results on the WEGA Stellarator
}

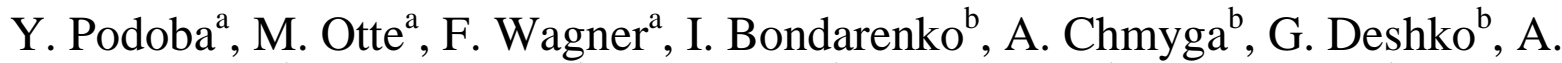 \\ Komarov $^{\mathrm{b}}$, A. Kozachek ${ }^{\mathrm{b}}$, L. Krupnik ${ }^{\mathrm{b}}, \mathrm{S}$. Khrebtov ${ }^{\mathrm{b}}$, A. Zhezhera ${ }^{\mathrm{b}}$, A. \\ Melnikov $^{\mathrm{c}}$, S. Perfilov ${ }^{\mathrm{c}}$, M. Schubert ${ }^{\mathrm{d}}$. \\ ${ }^{a}$ Max-Planck-Institut für Plasmaphysik, EURATOM Association, TI Greifswald, D-17491 Greifswald, Germany \\ ${ }^{b}$ Kharkov Institute of Plasma Physics, Kharkov, Ukraine \\ "INF RRC "Kurchatov Institute", Moscow, Russia. \\ ${ }^{d}$ L.P.M.I.A. Boulevard des Aiguillettes B.P. 239 54506, Vandoeuvre-Les-Nancy cedex, France.
}

\begin{abstract}
The heavy ion beam probe (HIBP) is an established non perturbing diagnostic for measuring the spatial distributions of plasma potential, density, temperature and poloidal magnetic field (axial current) of magnetically confined fusion plasma. These are determined from the change in the primary ion beam parameters (charge, intensity and trajectory) passing through a plasma volume due to collisions with electrons and interactions with the confining magnetic field. A heavy ion beam probe plasma diagnostic system has been installed and tested on the WEGA stellarator in Greifswald, Germany in 2006-2007. The WEGA HIBP operates with a beam of singly charged sodium ions with an energy of up to $60 \mathrm{keV}$, ion current up to $100 \mu \mathrm{A}$, and beam diameter of 5-6 mm in the confined plasma region. Plasma experiments with the HIBP diagnostic system were carried out at a magnetic field strength of $\mathrm{B}_{0}=0.489 \mathrm{~T}$. In the experiments, an argon plasma was heated with ECRH at $28 \mathrm{GHz}$. In this work the first plasma potential and total current profile measurements results are presented. This work was supported by STCU P-202 project.
\end{abstract}

Keywords: Stellarator, WEGA, HIBP, diagnostic development, plasma potential

PACS: 52.25.-b, 52.25.Tx, 52.25.Xz, 52.55.Hc, 52.65.Cc, 52.70.-m, 52.70.Nc

\section{INTRODUCTION}

The detailed knowledge of the electric field structure and its fluctuations from the core to the edge of the plasma is fundamentally important for understanding of the plasma confinement phenomena.

A Heavy Ion Beam Probe (HIBP) is a unique diagnostic, which is able to provide direct plasma electric potential measurements and its fluctuations from centre to the periphery of the plasma column.

The diagnostic is based on the injection of the singly charged ion beam across the confinement magnetic field. Some probing particles are ionized in the plasma and produce mainly doubly ionized secondary ions. The information about plasma parameters can be obtained from their characteristics. So, plasma potential obtained by this diagnostics is the difference between primary and secondary beam energy.

HIBP can be used in magnetic confinement fusion experiments to measure the electric potential $\Phi_{\mathrm{p}}$, electron density $n_{e}$ and electron temperature $T_{e}$, poloidal magnetic field $B_{\theta}$ as well as their fluctuations with a high temporal $(1 \div 10 \mu \mathrm{s})$ and spatial $(0.5 \div 1 \mathrm{~cm})$ resolution.

The HIBP diagnostic has been installed and tested on WEGA stellarator in collaboration with the Institute for Plasma Physics in Kharkov/Ukraine. The main objectives are the measurements for $28 \mathrm{GHz}$ ECR heated plasmas at a magnetic field strength of $\mathrm{B}=0.5 \mathrm{~T}$ of electric potential profiles, their validation with Langmuir probe data, and their comparison with the theoretical predictions for a classical stellarator.

\section{DESCRIPTION OF THE HIBP SYSTEM}

The $\mathrm{Na}^{+}$primary ion beam is used with an energy of $39.5 \mathrm{keV}$, current up to $50 \mu \mathrm{A}$ and beam width $\sim 5 \mathrm{~mm}$. Nominal toroidal magnetic field value in WEGA is $0.489 \mathrm{~T}$. The spatial resolution of $5 \mathrm{~mm}$ can be obtained along the detector line (or radial scan). The temporal resolution is $20 \mu \mathrm{S}$ and is limited mostly by the current to voltage converter bandwidth. The covered radial range of the measurements is $0.4<\mathrm{r} / \mathrm{a}<1$, unfortunately the plasma centre $(\mathrm{r} / \mathrm{a}=0)$ is not accessible due to geometrical limitations. 


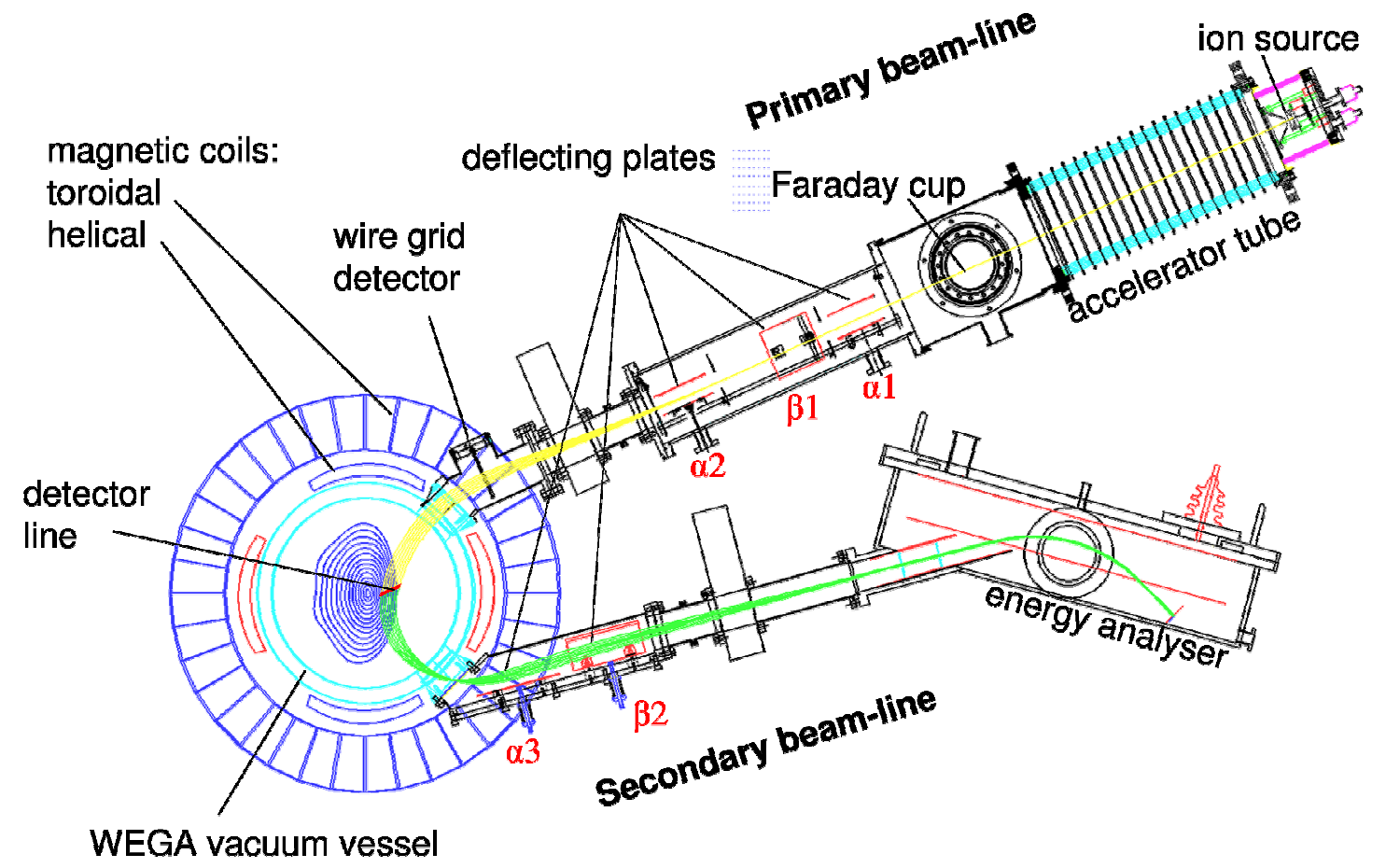

FIGURE 1. Schematic of the HIBP on the WEGA stellarator.

The HIBP system consists of two main parts (Fig.1): the ion beam injector with primary beam-line and the secondary beam-line with the energy analyser. The primary beam is emitted from a solid-state thermoemitter ion source. The $\mathrm{Na}^{+}$ions are emitted from a tablet, which is heated by a tungsten filament and then gain energy in the accelerating tube. Here ions are focused into a beam of about $5 \mathrm{~mm}$ diameter.

The beam current is measured by a Faraday cup installed after the accelerating tube. Typically, a beam current of about $50 \mu \mathrm{A}$ is used in the measurements but a current up to $100 \mu \mathrm{A}$ could be obtained on this system.

During the measurements, the Faraday cup lid is opened and the beam passes to a primary beam-line system, consisting of $\alpha 1, \alpha 2, \beta 1$ electrostatic deflecting plates. The sample volume position along the detector line in the plasma (see Fig.4a) is controlled by $\alpha 2$ plates voltage, while the $\alpha 3$ and $\beta 2$ plates in the secondary beam-line direct the double-charged particles from the ionization zone to the entrance slit of the detector.

After passing the deflection system, the beam reaches the wire grid detector. Here the beam width and shape can be detected. The current is measured on horizontal or on vertical wires when the beam crosses these wires during the $\alpha 2$ or $\beta 1$ scan correspondingly. Using linear voltage scan and known distance between wires, the beam width could be easily calculated. On the Fig.2a the measured current profiles are shown together with the $\alpha 2$ deflecting voltage. The measurements show the beam width of $\sim 5 \mathrm{~mm}$, which provides the spatial resolution of the plasma parameters measurements about $5 \mathrm{~mm}$.
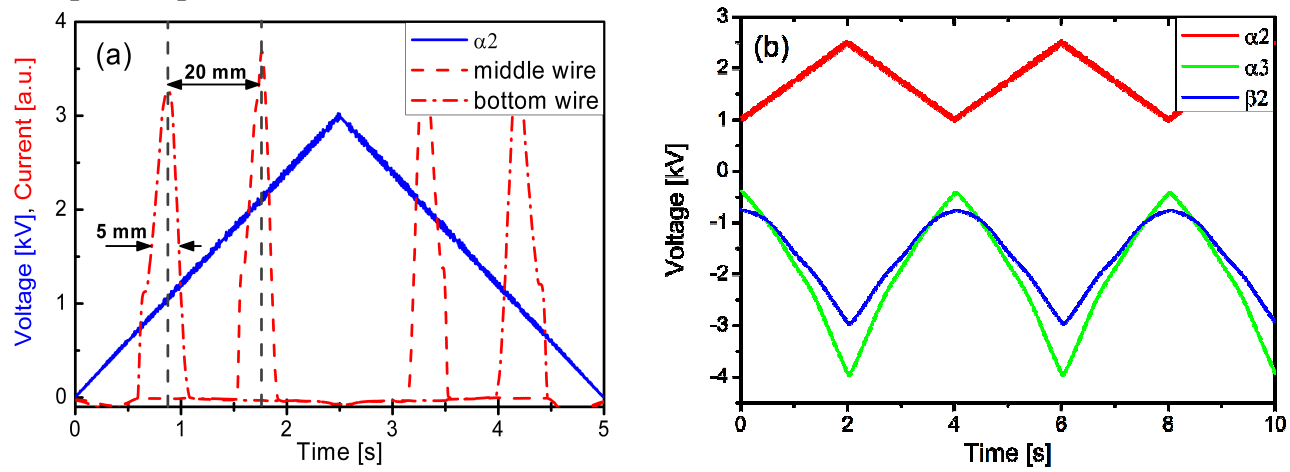

FIGURE 2. a) Currents measured by the horizontal wires of wire grid detector. Dashed and dot-dashed curves are currents measured on bottom and top wires correspondingly. Solid is the $\alpha 2$ control voltage. b) Synchronisation of $\alpha 3$ and $\beta 2$ control voltages with scanning $\alpha 2$ control voltage.

The wire grid detector is the last component of the primary beam line. After the wire grid detector the beam reaches the plasma where $\mathrm{Na}^{+}$ions are ionised and became double-charged $\mathrm{Na}^{++}$. Some of them, which originate in the sample volume, can reach the energy analyser. The energy of the double ionised ion is changed according 
to a potential in the ionisation point. If plasma potential is positive the ion gains energy equal to the potential in ionization point or looses the corresponding energy if the potential is negative.

The secondary beam-line includes two parts. First part is the system of secondary deflecting plates $\alpha 3$ and $\beta 2$. The voltages on these plates define the position of the sample volume along the primary beam trajectory. The energy analyser can detect the double charged ions from this volume. Thus, the voltages on these plates define the position and shape of the whole detector line during the profile scan and should be synchronized with the $\alpha 2$ voltage in such way to obtain the best coverage of the plasma by measurements. The typical behaviour of the $\alpha 2, \alpha 3$ and $\beta 2$ control voltages is shown in Fig.2b. The detector line shown in Fig.4a is obtained using this voltage synchronisation.

The Proca-Green design [2] energy analyser is used for the measurement of the secondary ions energy. In this type of analyzer, the energy of the ions is calculated from the deflection of the ions in a static electric field. In Fig.3a the scheme of the analyzer is shown. The beam enters the analyzer through the slit after the targeting plates that are used for beam position adjustment. Then it is deflected by the uniform electric field and hits four plates on which the current is measured. Using the relation of measured currents, the beam energy $\mathrm{W}_{\mathrm{s}}$ could be calculated.

$$
W_{s}=q_{s} V_{a}\left[G+F\left(i_{U}-i_{D}\right) /\left(i_{U}+i_{D}\right)\right] \text {, }
$$

where $\mathrm{G}$ and $\mathrm{F}-$ design parameters, $\mathrm{i}_{\mathrm{U}}=\mathrm{i}_{\mathrm{UL}}+\mathrm{i}_{\mathrm{UR}}, \mathrm{i}_{\mathrm{D}}=\mathrm{i}_{\mathrm{DL}}+\mathrm{i}_{\mathrm{DR}}$, currents measured on corresponding plates (Fig. $3 \mathrm{~b}), \mathrm{q}_{\mathrm{s}}$ charge of secondary ions, $\mathrm{V}_{\mathrm{a}}$ is the voltage applied to the deflecting plate of analyzer.

(a)

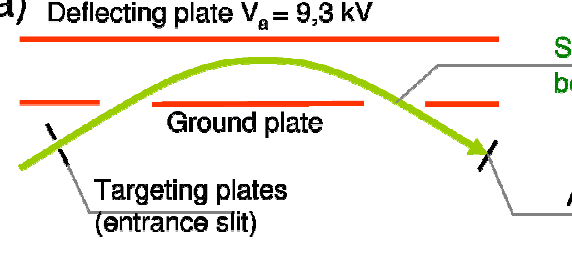

(b)

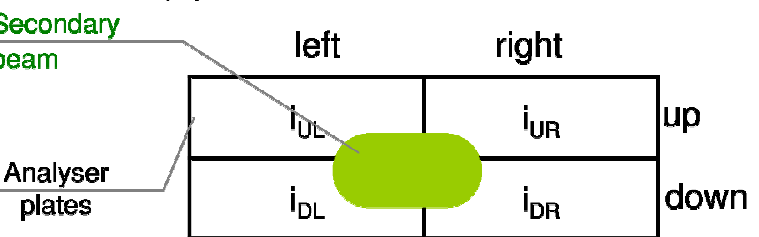

FIGURE 3. a) Energy analyser scheme, b) the analyser plates seen along the secondary beam trajectory.

\section{FIRST PROFILE}

The potential $\Phi_{\mathrm{p}}$ in the ionization point is calculated as a difference of the energy of primary and secondary beam. The latest result of the potential profile measurements is presented on the top of Fig. $4 \mathrm{~b}$. Here the potential is plotted over the voltage on the $\alpha 2$ deflecting plates. This corresponds to the scanning of the plasma potential along the detector line shown in Fig. 4a. As was mentioned the measurements cover the radial range of $0.4<$ r/a $<1$. The lower deflecting voltages correspond to a position closer to the edge of plasma.

(a)

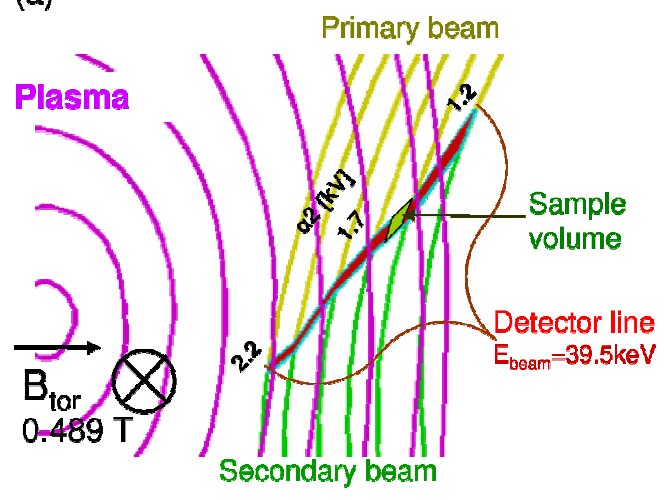

(b)

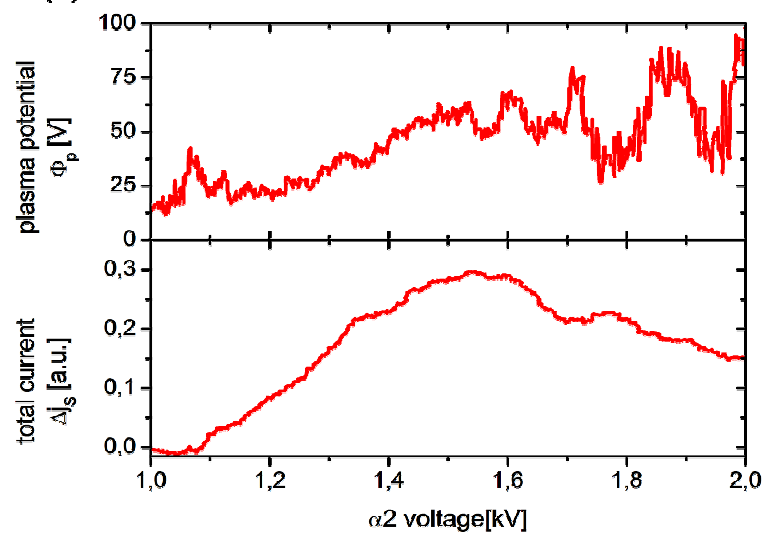

FIGURE 4. a) Detector line obtained from ray tracing calculations of primary and secondary beams. b) Plasma potential profile measured in ionization zone as shown in (a)

Potential measurement results indicate a positive plasma potential, using the vacuum vessel as the potential reference. This is in reasonable agreement with the data from Langmuir probes.

In addition, the total current of secondary ions $j_{s}$ was measured. This current is proportional to ionization cross-section $\sigma_{(i \rightarrow S)}$ and plasma density $n_{e}$ and could be calculated as

$$
j_{s}=\gamma_{e} \sigma_{(i \rightarrow S)} n_{e} l_{S V} F_{i} F_{S} j_{i} q_{s} / q_{i}
$$


where $\gamma_{e}$ is the secondary electron emission from detector plates, $l_{S V}$ is the length of the sample volume $\sim 5 \mathrm{~mm}, F$ is the beam attenuation factor. $F_{\mathrm{i}}, F_{S} \sim 1, j_{i}, j_{s}$ primary and secondary current density. The ionization cross-section is the function of the electron temperature. Thus, the full current is proportional to the plasma density if the electron temperature is constant. At the bottom plot of Fig.4b the total current measured along the same detector line as plasma potential is shown.

\section{CONCLUSIONS \& OUTLOOK}

The HIBP diagnostic is installed and tested on the WEGA stellarator. The parameters of the beam are improved which allows to increase the spatial resolution. First results show the good agreement of measured plasma potential with the data obtained from Langmuir probes measurements.

In the near future, the HIBP on the WEGA is planned to be used in experiments with power modulation of the $28 \mathrm{GHZ}$ ECRH to investigate the power deposition profile.

\section{ACKNOWLEDGMENTS}

The authors gratefully acknowledge the work of D. Aßmus, N. Paschkowski and R. Gerhardt for their technical support.

\section{REFERENCES}

1. L. I. Krupnik et al., Fusion Science and Technology 50, (2006) 276-280

2. T.S.Green and G.A.Proca, Rev.Scientific Instr. 41, 1409 (1970) 